Published in final edited form as:

J Am Acad Child Adolesc Psychiatry. 2010 January ; 49(1): 52-60.

\title{
Neurocognition in Early-Onset Schizophrenia and Schizoaffective Disorders
}

\author{
Dr. Stephen R. Hooper, Ph.D., Dr. Anthony J. Giuliano, Ph.D., Dr. Eric A. Youngstrom, \\ Ph.D., Dr. David Breiger, Ph.D., Dr. Linmarie Sikich, M.D., Dr. Jean A. Frazier, M.D., Dr. \\ Robert L. Findling, M.D., Dr. Jon McClellan, M.D., Dr. Robert M. Hamer, Ph.D., Dr. \\ Benedetto Vitiello, M.D., and Dr. Jeffrey A. Lieberman, M.D. \\ Drs. Hooper, Hamer, Sikich, and Youngstrom are with the University of North Carolina; Drs. \\ Giuliano and Frazier are with the Cambridge Health Alliance, Harvard Medical School; Drs. \\ Breiger and McClellan are with the University of Washington; Dr. Findling is with Case Western \\ Reserve University; Dr. Vitiello is with the National Institutes of Mental Health; Dr. Lieberman is \\ with Columbia University and New York State Psychiatric Institute
}

\begin{abstract}
Objective-We examined the neuropsychological functioning of youth enrolled in the NIMH funded trial, Treatment of Early-Onset Schizophrenia Spectrum Disorders (TEOSS). We compared the baseline neuropsychological functioning of youth with schizophrenia $(\mathrm{SZ}, \mathrm{n}=79)$ to those with schizoaffective disorder (SA, $n=40$ ), and examined the relationship of different variables of illness severity and adaptive behavior to neuropsychological functioning.
\end{abstract}

(O2010 American Academy of Child and Adolescent Psychiatry

Correspondence to Dr. Stephen R. Hooper, Center for Development and Learning, CB\# 7255, The Carolina Institute for Developmental Disabilities, University of North Carolina School of Medicine, Chapel Hill, NC 27599-7255; Stephen. hooper@cdl.unc.edu.

The opinions and assertions contained in this report are the private views of the authors and are not to be construed as official or as reflecting the views of the Department of Health and Human Services, the National Institutes of Health, or the National Institute of Mental Health

Disclosure: Dr. Hooper receives or has received research support or served as a speaker/consultant for Eli Lilly and Sanofi-Aventis. Dr. Youngstrom has previously served on the Data Safety and Monitoring Board for Eli Lilly and consulted in the past with Otsuka. Dr. Sikich has a current financial interest and receives research funding or participates in clinical trials with Janssen, Pfizer, Bristol Myers-Squibb, Neuropharm, Curemark, and Seaside Pharmaceuticals. She also received software for a computer intervention in schizophrenia from Posit Science. In the past, Dr. Sikich received research funding from Eli Lilly, Janssen, Pfizer, Otsuka, and Astra Zeneca, and has served as a consultant for Sanofi Aventis and ABT Associates. Dr. Frazier receives or has received research support and acted as a consultant for Bristol-Myers Squibb Company, Eli Lilly, GlaxoSmithKline, Johnson \& Johnson, Neuropharm, Otsuka America Pharmaceutical, and Pfizer. Dr. Findling receives or has received research support, acted as a consultant, and/or served on a speaker's bureau for Abbott, Addrenex, AstraZeneca, Biovail, Bristol-Myers Squibb, Forest, GlaxoSmithKline, Johnson \& Johnson, KemPharm, Eli Lilly, Lundbeck, Neuropharm, Novartis, Organon, Otsuka, Pfizer, Sanofi-Aventis, Sepracore, Shire, Solvay, Supernus Pharmaceuticals, Validus, and Wyeth. Dr. McClellan has had past research support from Pfizer. Dr. Hamer has served as a consultant or advisor for Acadia, Cenerx, Corcept, Epix, Johnson \& Johnson, NeuroPharmaBoost, Pepper-Hamil-ton, PureTechVentures, SanofiAventis, and Takeda. He has served on the advisory board for Enabled MD, Novartis, and Wyeth. He has served on a Data Safety Monitoring Board (DSMB) for Allergan, Eli Lilly \& Company, Pfizer, Schwartz, and Solvey. He has served on a Mock Advisory Panel for Alpharma. He has served as a statistician on a University of North Carolina contract for a clinical trial with AstraZeneca. He has taught several seminars for SAS Institute. He has served as an expert witness for Winston-Strawn. He or his wife own stock in Bristol-Myers Squibb, Amgen, Eli Lilly \& Company, Genentech, Proctor \& Gamble, and Sepracor. Dr. Lieberman serves as a consultant and/or advisor for Astra Zeneca, Bioline, Eli Lilly, Forest Laboratories, Glaxo-SmithKline, Pfizer, and Wyeth; and as a member of the Data Safety Monitoring Board (DSMB) for Solvay. He does not receive financial compensation or salary support for this participation as a consultant or as a member of a board. He receives grant support from AstraZeneca, Allon, Bristol-Myers Squibb, Cephalon, GlaxoSmithKline, Janssen, Merck, Pfizer, and Wyeth; and he holds a patent from Repligen. Drs. Guiliano, Breiger, and Vitiello report no biomedical financial interests or potential conflicts of interest. 
Method-Participants ranged in age from 8 to 19 years. Diagnostic status was confirmed via structured interview over multiple time points. Domains of neuropsychological functioning included fine-motor, attention, working memory, problem-solving efficiency, inhibitory control, and social cognition. Other variables included intelligence (IQ), academic achievement skills, adaptive behavior, and different measures of illness severity.

Results-The two groups did not differ on IQ or on any of the neuropsychological domains. The SZ group performed significantly lower in spelling. A high proportion of individuals in both groups reflected significant intellectual and academic achievement skill deficits. Significant correlations were found between the neurocognitive domains and both illness severity and adaptive behavior variables.

Conclusions-There were few differences between the SZ and SA groups on IQ, achievement, or neuropsychological functioning; however, both groups showed significantly high rates of deficits in IQ and basic academic skills. Correlations of the neurocognitive functions with illness severity and adaptive behavior were small to moderate in magnitude. These findings continue to implicate the importance of neurocognitive functioning as a key area of vulnerability in the study of youth with schizophrenia spectrum disorders.

\section{Keywords}

early-onset schizophrenia; childhood schizophrenia; schizoaffective disorder in childhood; neurocognition in schizophrenia

Although evidence has emerged over the past 15 to 20 years related to the neurocognitive features of early-onset schizophrenia spectrum disorders, ${ }^{1-7}$ debate continues as to whether schizophrenia (SZ) and schizoaffective (SA) disorder are continuous or distinct conditions. ${ }^{8-10}$ Among the many issues that represent points of controversy is the level and pattern of neurocognitive deficit associated with each disorder. ${ }^{11}$ Mixed findings are reported in the limited research that has been conducted with adult samples, ${ }^{12}$ and the issue has not been carefully examined in children and adolescents. The Treatment of Early Onset Schizophrenia Spectrum (TEOSS) study provides an opportunity to compare the level and pattern of neurocognitive functioning in a large sample of youth with onset of SZ or SA prior to 18 years of age.

Studies of adults suggest that the cognitive and functional outcomes of SA are intermediate between those with SZ (who have lower functioning) and those with depression or bipolar disorder alone (who have higher functioning) ${ }^{13-16}$ Individuals with the bipolar subtype of SA appear to have better outcomes than individuals with the depressive subtype. In the few studies examining neurocognition, memory impairments are reported to be greater in SZ than in SA groups. ${ }^{16,17}$ Apart from emerging evidence suggesting that adults with SA perform better on measures of social cognition, there are few, if any, additional reliable differences in cognitive functioning between these diagnostic groups. ${ }^{18,19}$

In the available studies of youth with early-onset schizophrenia spectrum disorders (EOSS), children and adolescents have evidenced generalized neurocognitive deficits compared with healthy community controls. ${ }^{4-6,20}$ However, when youth with EOSS are compared with groups with other psychotic illnesses (e.g., bipolar disorder, psychosis NOS), no significant 
group differences have been documented. ${ }^{21}$ Further, there is no published research comparing the level and pattern of neurocognitive functioning of youths with SZ or SA.

To this end, we examine the baseline neurocognitive characteristics of youths with SZ or SA participating in the TEOSS study. The analyses complement a prior publication on this same sample that compared the clinical and functional characteristics of children and adolescents with SZ or SA. ${ }^{21}$ In that paper, we reported similar levels of clinical symptoms and functioning across domains between the two diagnostic groups. ${ }^{17}$ Taken together with the limited adult literature, we hypothesized that group comparisons on neurocognitive functioning would yield more similarities than differences.

\section{METHOD}

\section{Participants}

Participants included 119 children and adolescents ranging in age from 8.1 to 19.3 years (mean $=14.25$ years, $\mathrm{SD}=2.41$ ). The sample was approximately two-thirds male, $61.4 \%$ white and $88.3 \%$ right handed, and fell within the low-middle socioeconomic strata as determined by family income. Approrximately $45 \%$ of the sample was receiving special education services at the time of enrollment. Approximately $53 \%$ had a previous psychiatric hospitalization, and 56\% had previously received pharmacological treatment for mood and/or behavioral difficulties. At baseline, approximately $68 \%$ were receiving some type of psychiatric medication; however, none were receiving any anti-psychotic pharmacological agents for their schizophrenia spectrum difficulties. Only 11 of the participants had engaged in illicit substance usage. Inclusion criteria included meeting DSM-IV 22 criteria for schizophrenia, schizophreniform disorder, or schizoaffective disorder, via the Structured Clinical Interview for DSM-IV Childhood Disorders (KID-SCID), ${ }^{23}$ and a score of at least moderate on one or more of the positive psychotic symptoms of the Positive and Negative Syndrome Scale (PANSS). Interrater reliability within and between sites was maintained at $\searrow 0.80$.

The KID-SCID was selected because of its greater sensitivity to psychotic symptomatology and clear diagnostic algorithms for determining SA versus SZ. Furthermore, all diagnoses were reassessed upon termination of study participations using the KID-SCID in an effort to examine severity and duration of mood symptoms relative to psychotic symptoms. Early diagnoses were found to be stable, with only one participant's diagnosis changing from SZ at baseline to SA at study endpoint. All clinicians were fully trained general psychiatrists, and many had participated in adult studies in which psychotic symptoms and diagnoses were evaluated. The TEOSS protocol was approved by the Institutional Review Boards at all four participating sites. A more complete description of TEOSS methods is published elsewhere. ${ }^{24}$

\section{Measures}

The battery of tasks assessed targeted neurocognitive domains: fine-motor speed (Manual Finger Tapping Test, ${ }^{25}$ Grooved Pegboard ${ }^{26}$ ) attention and inhibitory control (VIGIL Continuous Performance Test ${ }^{27}$ ), working memory (Hopkins Verbal Learning Test, ${ }^{28}$ Wide 
Range Assessment of Memory and Learning Visual Learning Subtest, ${ }^{29}$ Visuospatial Working Memory Test, ${ }^{30}$ Woodcock-Johnson-III Numbers Reversed Subtest ${ }^{31}$ ), problemsolving efficiency (Controlled Oral Word Association-Semantic, ${ }^{32}$ Ruff Figural Fluency, ${ }^{33}$ Wisconsin Card Sorting Test-64 ${ }^{34}$ ), and social cognition (Eyes Test ${ }^{35}$ ). Psychoeducational tasks measured intelligence (Wechsler Abbreviated Scales of Intelligence ${ }^{36}$ ) and basic academic achievement skills (Wide Range Achievement Test $-3^{37}$ ). There was coordination with some measurement in the Clinical Antipsychotic Trials of Intervention Effectiveness (CATIE) study that was examining similar questions in older adolescents and adults. ${ }^{38}$

Measures were blocked and the blocks counterbalanced to control for order effects. The blocks were constructed such that at least one measure represented each of the neurocognitive domains so that we could obtain information on a specific domain in the event that a participant was unable to continue for any reason. In addition, a number of measures were used to address symptom severity and functional status including the Positive and Negative Symptom Scale (PANSS), ${ }^{39}$ Clinical Global Impressions Scale (CGI) ${ }^{40}$ Brief Psychiatric Rating Scale for Children (BPRS-C), ${ }^{41}$ Child Behavior Checklist (CBCL), ${ }^{42}$ and Vineland Adaptive Behavior Scale (VABS). ${ }^{43}$

\section{Data Analyses}

Preliminary data analyses compared the SZ and the SA groups on demographic and illnessrelated variables. Any variables found to be significantly different between the groups were considered as covariates in subsequent analyses.

Using univariate analyses of variance, we examined group differences on the psychoeducational measures (IQ, achievement). For any significant group differences, effect sizes were calculated using Cohen's $d$ statistic. In addition, given that the WASI and WRAT-3 are well normed, we examined the percentage of participants falling $\geq 1$ standard deviation (SD) below each of these tests' means as another clinical index of functioning.

For the neurocognitive tasks we created sample-based $z$-scores using the entire sample of EOSS cases. ${ }^{44}$ All $z$-scores were scaled such that higher scores reflected a better performance with respect to the overall sample mean. Using these $z$-scores, we then created neurocognitive domain scores and calculated alpha coefficients for each domain: FineMotor (Fingertapping Dominant + Fingertapping Nondominant + Grooved Pegboard Dominant + Grooved Pegboard Nondominant), Attention (Visual CPT Omission Errors + Auditory CPT Omission Errors), Working Memory (HVLT Total + WRAML Total + Visual-Spatial 5' Delay + Numbers Reversed), Problem Solving Efficiency (WCST Categories + WCST Perseverations + COWA Semantic Fluency + Ruff Unique Designs), Inhibitory Control (Visual CPT Commission Errors + Auditory CPT Commission Errors), and Social Cognition (Eyes Test Total Correct). Using General Linear Modeling, we conducted a series of univariate analyses across the two groups on the neurocognitive domains and their associated tasks controlling for chronological age (because several of the tests were administered out of normative range) and any other demographic variables that may have been different between the groups. For any significant group differences, effect sizes were calculated using Cohen's d statistic. 
Finally, we conducted Pearson correlations, partial-ling out chronological age, between the neurocognitive domains and symptom severity as defined by scores from the PANSS, BPRS-C, CGI, and CBCL, and selected illness-related variables (illness duration, age of onset). A similar analysis was conducted between the neurocognitive domains and the VABS.

\section{RESULTS}

\section{Preliminary Group Comparisons}

Of the 119 participants in this study, $89.9 \%$ of the participants were able to complete some of the tests in the neuropsychological assessment battery. Given our blocking strategy, completion rates ranged from $80.0 \%$ for the Social Cognition Domain to $89.0 \%$ for the Problem Solving Efficiency Domain. Eleven participants were unable or unwilling to complete any of the neuropsychological tasks at baseline. When these individuals were compared with the rest of the sample, no differences were noted for age, gender, race, or family income. With respect to illness-related variables, these groups did not differ on the number of previous psychiatric hospitalizations, prior medication use, age at first psychosis, duration of illness, CGI, BPRS-C Total Summary Score, PANSS Positive Symptoms, PANSS Negative Symptoms, or PANSS Total Summary.

Descriptive information characterizing the sample is provided in Table 1. The two diagnostic groups did not differ on chronological age, gender, race, or handedness. The SA group had significantly higher family income than the SZ group, $t(109)=-2.05, p<.04$. With respect to illness-related variables, the two groups did not differ on the number of previous psychiatric hospitalizations, prior medication use, age at first psychosis, duration of illness, special education services, PANSS Summary score, PANSS Positive Symptoms Scale, BPRS-C, or the CGI. The groups differed on the PANSS Negative Symptoms Scale, $t$ $(115)=2.30, p<.02$, with the SZ group having more negative symptoms.

\section{Intellectual and Academic Skills of Youth with SZ versus SA}

The overall intellectual functioning for the sample fell within the low-average to average range, with the verbal and performance IQs being evenly developed within that range (Table 2). Reading and spelling skills fell within a comparable range of functioning, although arithmetic skills were nearly $1 \mathrm{SD}$ below the normative mean.

The two groups did not differ in terms of their Verbal IQ, Performance IQ, Full Scale IQ, or arithmetic skills (Table 2); however, group differences approached significance on reading, $F(1,97)=2.71, p<.10$, and the groups were significantly different on spelling, $F(1,96)=$ $3.92, p<.05$. For both reading and spelling, the SA group performed at a higher level.

Percentage of Participants Falling $\geq 1$ SD Below the Mean-Given the relatively lower IQ and achievement scores, the possibility that a large number of participants were performing at a significantly lower level of functioning was examined. In this regard, we determined the percentage of participants who fell $\geq 1 \mathrm{SD}$ below the normative mean (i.e., standard score $\$ 85$ ). For IQ, the percentage of participants who fell $\geq 1 \mathrm{SD}$ below the mean ranged from about 32\% (Performance IQ) to nearly 39\% (Full Scale IQ). For academic 
achievement, these percentages ranged from approximately $22 \%$ (WRAT-3 Reading) to approximately 51\% (WRAT-3 Arithmetic). Taken together, these percentages reveal that a high proportion of the entire sample is functioning below average on indices of IQ and academic achievement skills. Despite the relatively large number of individuals that met this criterion in the overall sample, there were no significant differences between the percentages represented in each of the diagnostic groups.

\section{Neurocognitive Functioning of SZ versus SA}

Once sample-specific $z$-scores for each of the neurocognitive tests and their associated domain were created, alpha coefficients for these domains were deemed to be satisfactory (ranging from 0.71 on Inhibitory Control to 0.85 for Fine-Motor Speed). In addition, all of the tasks had good floors and ceilings with this sample, so no range restrictions were noted. After controlling for age, there were no significant differences between the groups across the six neurocognitive domains or on any of the specific neurocognitive measures (Table 3). The results were relatively unchanged when both age and socioeconomic status were included as covariates.

\section{Relationship Between Neurocognitive Functions, Symptom Severity, and Adaptive Behavior}

Symptom Severity-After controlling for age, there were no significant correlations between the neurocognitive domains and the PANSS Positive, PANSS Negative, PANSS Summary, BPRS-C Total Summary, and the CBCL summary scales. Of the neurocognitive domains, only Full Scale IQ was significantly, but weakly, correlated with age of onset ( $r=$ -0.28 ) and duration of illness $(r=0.30)$. This implies that higher IQ was modestly associated with a younger age of onset and a longer duration of illness. The latter variable of illness duration may be an artifact of being diagnosed at a younger age and, indeed, these variables were highly correlated $(r=-0.68)$; however, this finding does not support the common notion that diagnosis at a younger age portends a more severe form of the disorder - at least not from a cognitive perspective. In fact, when we examined these relationships, there was little evidence for a significant association between clinician ratings of illness severity and age of onset $(r=0.14)$, or clinician ratings of illness severity and illness duration $(r=-0.04)$. This conceptualization is supported, in part, by the significant negative relationship between higher IQ being associated with better clinician ratings of illness severity on the CGI $(r=-0.27)$. This relationship could reflect an interesting artifact such that higher IQ (e.g., a highly verbal individual) may influence clinician ratings of illness severity in a positive direction. The most consistent pattern of correlations was observed for the CGI ratings at baseline, with correlations ranging from -0.24 (Attention) to -0.37 (Problem Solving Efficiency). For the CGI, better ratings of global impairment were associated with better attention and problem-solving capabilities.

Adaptive Behavior-Consistent with the adult literature, a more robust pattern of correlations was noted between the neurocognitive domains and adaptive behavior, as displayed in Table 4. The VABS Communication Skills, Daily Living Skills, and Social Skills were each significantly correlated with IQ and four or more of the seven neurocognitive domains. Correlations ranged from small $(r=0.21, p<.05$, between Social 
Cognition and VABS Communication) to moderate ( $r=0.45, p<.001$, between Working Memory and VABS Communication). Attention, Working Memory, and Problem Solving Efficiency had the most robust associations with adaptive behaviors. All of the correlations were in the expected direction (i.e., higher neurocognitive domain scores were associated with higher adaptive behavior skills).

Similar relationships were observed between nearly all of the neurocognitive domains and the adaptive behavior composite (Table 4). For the adaptive behavior composite, correlations ranged from small $(r=0.23, p<.01)$ for Social Cognition to moderate $(r=0.44$, $p<.001)$ for Working Memory. Inhibitory Control did not significantly correlate with the Vineland Adaptive Behavior Composite, but it approached significance $(r=0.19, p<.07)$ in the expected direction.

\section{DISCUSSION}

We did not find significant differences between youth with SZ and those with SA disorder on any of the neuropsychological variables. The only significant group difference was obtained within the academic skill domain, in which the SZ group performed significantly lower on a task of single word spelling. These results are consistent with our earlier findings ${ }^{17}$ that indicated few clinical differences between these groups. Although some studies of adults suggest that individuals with SZ have greater cognitive impairment than those with $\mathrm{SA},{ }^{45}$ the available evidence also suggests a lack of differentiation between these two disorders in adults. ${ }^{18,19,46}$ These findings are inconsistent with the theory that SA disorder might represent the juxtaposition of two different illnesses, or a more severe variant of schizophrenia, as both of these models would instead predict the greatest cumulative deficits for SA versus "pure" SZ cases.

A substantial proportion of participants across both diagnostic groups demonstrated clinically significant intellectual impairment and academic delays. Approximately one-third of the sample was $\geq 1$ SD below the normative mean in their Verbal, Performance, or Full Scale IQ scores. The greatest academic deficits were in arithmetic, in which more than half of the entire sample was $\geq 1 \mathrm{SD}$ below the mean. Nearly one-quarter of the sample also was below average in reading and spelling. Although neurologically based explanations (e.g., right hemisphere involvement, efficiency of white matter tracts) and/or neurodevelopmental models (e.g., onset is preceded by a more vulnerable premorbid period marked by lower $\mathrm{IQ}^{47}$ ) could be invoked to explain why math skills were significantly lower for this group when compared with normative expectations, a more parsimonious explanation is that school-related learning experiences are less available to these children because of the psychological limitations imposed by the illness. Further, the combination of cognitive deficiencies and serious psychiatric difficulties may result in limited cognitive resources available for other adaptive functions, such as learning hierarchical information found in mathematics or the acquisition and developmental progression of reading and spelling skills. Taken together, these deficits will place many children with schizophrenia spectrum disorders at risk for school failure. 
The CGI ratings of illness severity at baseline provided the most extensive correlations with neurocognitive functioning, with less symptom severity being related to higher neurocognitive performance. Similarly, we found the social, communication, and daily living adaptive behavior skills, as well as overall adaptive behavior functioning, to be significantly correlated with nearly all of the neuropsychological domains. These significant relationships are not surprising, and reflect that more severely ill youth typically have greater impairments in multiple domains. The magnitude of these associations is moderate, and becomes even more impressive when considering that the correlations are not inflated by shared method variance. ${ }^{48}$ Similar associations have been reported in the adult literature. ${ }^{49,50}$ These results strengthen the notion that at least some of the burden of SZrelated disorders may be due to impaired neurocognitive functioning.

One curious finding relates to the significant relationship between Full Scale IQ and age of onset of the disorder, where higher IQ was associated with a younger age of onset. This finding does not support the notion that diagnosis at an early age suggests a more severe form of the disorder but perhaps instead a milder variant, as suggested by the modestly significant and negative relationship between IQ and ratings of illness severity. In addition to the potential artifacts noted above (e.g., a verbal individual may influence positive ratings even when showing heightened illness severity), this finding will require replication and elucidation in the context of the potential effects of duration of untreated psychosis (i.e., it is possible that illness onset at a younger age increases the likelihood of early detection and treatment, shortening the duration of untreated psychosis and attenuating progressive pathological processes) or youth at imminent risk for violence or suicide-variables that were not measured in TEOSS. Further, the range of ages of onset in our study was restricted by the inclusion criteria (i.e., all of our subjects had an early-onset form of illness). Taken together, the generalizability of this finding to community-based samples may be lessened. In addition, although the use of illicit substances was not a factor for either of the clinical groups, these substances could have clear implications for the manifestation of psychotic illness and for gene-environment interactions in the onset of psychosis in later studies. ${ }^{51}$

Positive symptoms did not correlate with neurocognitive domain scores, which is consistent with studies of adults with first-episode and chronic SZ; ${ }^{45,52-54}$ but neither did negative symptoms. Across the adult studies that show a significant relationship between negative symptoms and cognitive dysfunction, the correlations typically are small in magnitude and account for a minor portion of the variance (often $10 \%$ to $15 \%$ ). ${ }^{45,52-54}$ Even when the significant correlations of the neurocognitive domains with the CGI and adaptive behaviors obtained in this study are considered, the magnitude of these correlations accounted for only $6 \%$ to $14 \%$ of the variance, similar to the range reported in adult studies.

Finally, from an assessment perspective, our sample seemed to tolerate the measures used in this study. The next step is to determine whether this battery is sensitive to cognitive changes following pharmacological intervention. Consistent with the design of the MATRICS Consensus Battery, ${ }^{44}$ our measures were selected to detect changes in neurocognitive functioning secondary to medication effects. In that regard, our measurement does map onto many of the domains contained within the MATRICS Battery, such as Attention/Vigilance, Working Memory, Reasoning/Problem Solving, and Social Cognition. 
Our findings suggest that the selected tasks are appropriate for assessing the neurocognitive functioning in youth with EOSS. The associations between neurocognitive performance and adaptive behavior or global functioning reinforce the potential value of measuring neurocognition and, perhaps, regarding it as an important treatment target in its own right.

The primary purpose of this study was to examine the similarities and differences in neurocognitive performance in a well-characterized group of children and adolescents with $\mathrm{SZ}$ or SA. Our findings represent some of the first data examining this question in youth. We did not find any differences in the level of performance between the groups across the neurocognitive domains examined. For the larger sample, severity of illness is associated with greater neurocognitive and adaptive functioning deficits, but this is neither surprising nor diagnostically specific. The lack of a definitive neurocognitive profile unique to any given psychotic disorder is a predictable outcome given substantial clinical and etiological heterogeneity within derived diagnostic categories. Recent findings suggest that schizophrenia spectrum disorders are characterized by marked genetic heterogeneity. ${ }^{55-57}$ These disorders appear to stem from a large number of different overlapping disruptions in key neurodevelopmental pathways. As these critical pathways are identified, neurocognitive research can focus on the impact of disruptions in specific brain networks, rather than on global findings shared across groups of individuals with phenotypically related but heterogeneous syndromes. ${ }^{58}$

\section{Acknowledgments}

The Treatment of Early Onset Schizophrenia Spectrum (TEOSS) project was conducted with grant support from the National Institute of Mental Health under cooperative agreements U01MH61528 to the University of North Carolina (P.I.: Lin Sikich), U01MH61464 to the University of Washington (P.I.: Jon McClellan), U01MH62726 to Harvard Medical School (P.I.: Jean Frazier), and U01MH61355 to Case Western Reserve University (P.I.: Robert Findling), and by the Maternal Child Health Bureau (\#MCJ379154A), and the Administration on Developmental Disabilities (\#90DD043003). The research was conducted in NIH supported Clinical Research Centers at Seattle Children's Hospital, University of Washington (M01-RR-00037) and the University of North Carolina (M01RR00046).

We acknowledge the significant day-to-day contributions by the Research Associates: Kathryn DiFrancesco at Case Western Reserve; Leslie Pierson and Jennifer Varley at Seattle Children's Hospital; Emily Gerstein at Cambridge Health Alliance, Harvard Medical School; and Vicki Dixon, Cathy Jones, Andy Petesch, and Tywanda Lightfoot at the University of North Carolina School of Medicine.

\section{References}

1. Cornblatt B, Obuchowski M, Roberts S, Pollack S, Erlenmeyer-Kimling L. Cognitive and behavioral precursors of schizophrenia. Dev Psychopathol. 1999; 11:487-508. [PubMed: 10532621]

2. Iacono WG, Clementz BA. A strategy for elucidating genetic influence on complex psychopathological syndromes. Prog Exp Psychopath Res. 1993; 16:11-65.

3. Kumra S, Wiggs E, Bedwell J, et al. Neuropsychological deficits in pediatric patients with childhood-onset schizophrenia and psychotic disorder not otherwise specified. Schizophr Res. 2000; 7:135-144. [PubMed: 10742651]

4. McClelland J, Prezbindowski A, Breiger D, McCurry C. Neuropsychological functioning in earlyonset psychotic disorders. Schizophr Res. 2004; 28:21-29.

5. Rhinewine JP, Lencz T, Thaden EP, et al. Neurocognitive profile in adolescents with early-onset schizophrenia: clinical correlates. Biol Psychiatry. 2005; 58:705-712. [PubMed: 16023084]

6. Ueland T, Oie N, Landro I, Rund B. Cognitive functioning in adolescents with schizophrenia spectrum disorders. Psychiatry Res. 2004; 126:229-239. [PubMed: 15157749] 
7. Walker EF, Savoie T, Davis D. Neuromotor precursors of schizophrenia. Schizophr Bull. 1994; 20:453-480. [PubMed: 7526447]

8. Evans JD, Heaton RK, Paulsen JS, McAdams LA, Heaton SC, Jeste DV. Schizoaffective disorder: a form of schizophrenia or affective disorder. J Clin Psychiatry. 1999; 60:874-882. [PubMed: 10665641]

9. Lake CR, Hurwitz N. Schizoaffective disorders are psychotic mood disorders; there are no schizoaffective disorders. Psychiatry Res. 2006; 143:255-287. [PubMed: 16857267]

10. Malhi GS, Green M, Fagiolini A, Peselow ED, Kumari V. Schizoaffective disorder: diagnostic issues and future recommendations. Bipolar Disord. 2008; 10:215-230. [PubMed: 18199238]

11. Bearden CE, Freimer NB. Endophenotypes for psychiatric disorders: ready for primetime? Trends Genet. 2006; 22:306-310. [PubMed: 16697071]

12. Kempf L, Hussain N, Potash JB. Mood disorder with psychotic features, schizoaffective disorder, and schizophrenia with mood features: trouble at the borders. Int Rev Psychiatry. 2005; 17:9-19. [PubMed: 16194767]

13. Harrow M, Grossman LS, Herbener ES, Davies EW. Ten-year outcome: patients with schizoaffective disorders, schizophrenia, affective disorders and mood-incongruent psychotic symptoms. Br J Psychiatry. 2000; 177:421-426. [PubMed: 11059995]

14. Jager M, Bottlender R, Strauss A, Moller J. Fifteen-year follow-up of ICD-schizoaffective disorders compared with schizophrenia and affective disorders. Acta Psychiatr Scand. 2004; 109:117-121.

15. Reinares, M.; Vieta, E.; Benabarre, A.; Marneros, A. Clinical course of schizoaffective disorders. In: Marneros, A.; Akiskal, AH., editors. The Overlap of Affective and Schizophrenic Spectra. Cambridge, UK: Cambridge University Press; 2007. p. 145-155.

16. Beatty WW, Jocic Z, Monson N, Staton RD. Memory and frontal lobe dysfunction in schizophrenia and schizoaffective disorder. J Nerv Ment Dis. 1993; 181:448-453. [PubMed: 8320548]

17. Verdoux H, Liraud F. Neuropsychological function in subjects with psychotic and affective disorders: relationship to diagnostic category and duration of illness. Eur Psychiatry. 2000; 15:236-243. [PubMed: 10951607]

18. Addington J, Brooks BL, Addington D. Cognitive functioning in first episode psychosis: initial presentation. Schizophr Res. 2003; 62:59-64. [PubMed: 12765744]

19. Fiszdon JM, Richardson R, Greig T, Bell MD. A comparison of basic and social cognition between schizophrenia and schizoaffective disorder. Schizophr Res. 2007; 91:117-121. [PubMed: 17258431]

20. Thaden E, Rhinewine JP, Lencz T. Early-onset schizophrenia is associated with impaired adolescent development of attentional capacity using the Identical Pairs Continuous Performance Test. Schizophr Res. 2006; 81:157-166. [PubMed: 16309895]

21. Frazier JA, McClellan J, Findling RL, et al. Treatment of early-onset schizophrenia spectrum disorders (TEOSS): demographic and clinical characteristics. J Am Acad Child Adolesc Psychiatry. 2007; 46:979-988. [PubMed: 17667477]

22. American Psychiatric Association. Diagnostic and Statistical Manual-IV-TR. Washington, DC: American Psychiatric Association; 1994.

23. Hein, D.; Matzner, F.; First, M.; Spitzer, R.; Williams, J.; Gibbons, M. Structured clinical interview for DSM-IV childhood disorders, KID-SCID. New York, NY: Department of Psychiatry, Columbia University Medical School; 1998.

24. McClellan J, Sikich L, Findling RL, et al. Treatment of early-onset schizophrenia spectrum disorders (TEOSS). Rationale, design, and methods. J Am Acad Child Adolesc Psychiatry. 2007; 46:969-978. [PubMed: 17667476]

25. Reitan, RM.; Wolfson, D. The Halstead-Reitan Neuropsychological Test Battery: Theory and Interpretation. Tucson, AZ: Neuropsychology Press; 1985.

26. Lafayette Instrument Company. Grooved Pegboard Instruction Manual, Model 32025. Lafayette, IN: Lafayette Instrument Company; 1989.

27. Cegalis, J.; Bowlin, J. Vigil: Software for the Assessment of Attention. Nashua, NH: Forthought; 1991. 
28. Brandt J. The Hopkins Verbal Learning Test: development of a new verbal memory test with six equivalent forms. Clin Neuropsychol. 1991; 5:125-142.

29. Adams, W.; Sheslow, D. The Wide Range Assessment of Memory and Learning. Wilmington, DE: Jastak Associates; 1990.

30. Hershey T, Selke G, Fucetola R, Newcomer JW. Spatial long-term memory but not working memory decreases over time in schizophrenia [Abstract]. Soc Neuroscience Abs. 1999; 25:572.

31. Woodcock, RW.; McGrew, KS.; Mather, N. Woodcock-Johnson-III Tests of Cognitive Abilities. Chicago, IL: Riverside Publishing; 2001.

32. Benton, AL.; Hamscher, K. Multilingual Aphasia Examination Manual (revised). Iowa City, IA: University of Iowa; 1978.

33. Ruff RM, Light RH, Evans RW. The Ruff Figural Fluency Test: a normative study with adults. Dev Neuropsychol. 1987; 3:37-51.

34. Heaton, RK.; Chelune, GJ.; Taley, JL.; Kay, GG.; Curtiss, G. Wisconsin Card Sorting Test Manual: Revised and Expanded. Odessa, FL: Psychological Assessment Resources; 1993.

35. Baron-Cohen S, Wheelwright S, Hill J, Raste Y, Plumb I. The 'Reading the Mind in the Eyes' test revised version: a study with normal adults, and adults with Asperger syndrome or highfunctioning autism. J Child Psychol Psychiatry. 2001; 42:241-252. [PubMed: 11280420]

36. Stano, JF. Wechsler Abbreviated Scale of Intelligence. San Antonio, TX: Psychological Corporation; 1999.

37. Wilkenson, GS. Wide Range Achievement Test-Third Revision. Wilmington, DE: Jastak Associates; 1993.

38. Keefe RSE, Mohs RC, Bilder RM, et al. Neurocognitive assessment in the Clinical Antipsychotic Trials of Intervention Effectiveness (CATIE) project schizophrenia trial: development, methodology, and rationale. Schizophr Bull. 2003; 29:45-55. [PubMed: 12908660]

39. Kay S, Opler L, Lindenmayer J. The Positive and Negative Syndrome Scale (PANSS): rationale and standardisation. Br J Psychiatry. 1989; 155:59-67.

40. Guy, W. ECDEU Assessment Manual for Psychopharmacology. Washington, DC: National Institute of Mental Health; 1976. Clinical Global Impression; p. 217-221.

41. Overall J, Pfefferbaum B. The Brief Psychiatric Rating Scale for Children. Psychopharmacol Bull. 1982; 18:10-16. [PubMed: 7111598]

42. Achenbach, TM.; Rescorla, LA. Manual for ASEBA School-Age Forms \& Profiles. Burlington, VT: University of Vermont, Research Center for Children, Youth, \& Families; 2001.

43. Sparrow, SS.; Balla, DA.; Cicchetti, DV. Vineland Adaptive Behavior Scales, Survey Edition. Circle Pines, MN: American Guidance Service; 1984.

44. Hill SK, Sweeney JA, Hamer RM, et al. Efficiency of the CATIE and BACS neuropsychological batteries in assessing cognitive effects of antipsychotic treatments in schizophrenia. J Int Neuropsychol Soc. 2008; 14:209-221. [PubMed: 18282319]

45. Bilder RM, Goldman RS, Robinson D, et al. Neuropsychology of first-episode schizophrenia: initial characterization and clinical correlates. Am J Psychiatry. 2000; 157:549-559. [PubMed: 10739413]

46. Goldstein G, Shemansky WJ, Allen DN. Cognitive function in schizoaffective disorder and clinical subtypes of schizophrenia. Arch Clin Neuropsychol. 2005; 20:153-159. [PubMed: 15708725]

47. Woodberry KA, Giuliano AJ, Seidman LJ. Premorbid IQ in schizophrenia: a meta-analytic review. Am J Psychiatry. 2008; 165:579-587. [PubMed: 18413704]

48. Campbell DT, Fiske DW. Convergent and discriminant validation by the multitrait-multi-method matrix. Psych Bull. 1959; 56:81-105.

49. Green MF. What are the functional consequences of neurocognitive deficits in schizophrenia? Am J Psychiatry. 1996; 153:321-330. [PubMed: 8610818]

50. Semkovska M, Bedard M-A, Godbout L, Limoge F, Stip E. Assessment of executive dysfunction during activities of daily living in schizophrenia. Schizophr Res. 2004; 69:289-300. [PubMed: 15469200]

51. Henquet C, Di Forti M, Morrison P, Kuepper R, Murray RM. Gene-environment interplay between cannabis and psychosis. Schizophr Bull. 2008; 34:1111-1121. [PubMed: 18723841] 
52. Addington, J. Negative symptoms and the relationship to cognitive functioning. In: Sharma, T.; Harvey, P., editors. Cognition in Schizophrenia. Oxford, UK: Oxford University Press; 2000. p. 191-209.

53. Heydebrand G, Weiser M, Rabinowitz J, Hoff AL, DeLisi LE, Csernansky JG. Correlates of cognitive deficits in first episode schizophrenia. Schizophr Res. 2004; 68:1-9. [PubMed: 15037334]

54. Velligan DI, Mahurin RK, Diamond PL, Hazelton BC, Eckert SL, Miller AL. The functional significance of symptomatology and cognitive function in schizophrenia. Schizophr Res. 1997; 25:21-31. [PubMed: 9176924]

55. Walsh T, McClellan JM, McCarthy SE, et al. Rare structural variants disrupt multiple genes in neurodevelopmental pathways in schizophrenia. Science. 2008; 320:539-543. [PubMed: 18369103]

56. Xu B, Roos JL, Levy S, van Rensburg EJ, Gogos JA, Karayiorgou M. Strong association of de novo copy number mutations with sporadic schizophrenia. Nat Genet. 2008; 40:880-885. [PubMed: 18511947]

57. Stefansson H, Rujescu D, Cichon S, et al. Large recurrent microdeletions associated with schizophrenia. Nature. 2008; 455:232-236. [PubMed: 18668039]

58. Burdick KE, Goldberg JF, Harrow M, Faull RN, Malhorta AK. Neurocognition as a stable endophenotype in bipolar disorder and schizophrenia. J Nerv Ment Dis. 2006; 194:255-260. [PubMed: 16614546] 


\section{TABLE 1}

Demographics Characteristics for the Total Sample and Clinical Subsamples

\begin{tabular}{|c|c|c|c|}
\hline Characteristic & Total Sample $(\mathrm{N}=119)$ & Schizophrenia $(n=79)$ & Schizoaffective $(n=40)$ \\
\hline Chronological age (y) & $14.25(2.41)$ & $14.26(2.45)$ & $14.22(2.38)$ \\
\hline Gender ( $\%$ male) & $65.6 \%$ & 63.3 & $70.0 \%$ \\
\hline Race (\% white) & $61.4 \%$ & $57.0 \%$ & $70.0 \%$ \\
\hline Family income $a^{*}$ & $2.75(1.52)$ & $2.55(1.42)$ & $3.17(1.65)$ \\
\hline Handedness (\% Right) & $88.3 \%$ & $87.3 \%$ & $90.0 \%$ \\
\hline Special education (\%) & $45.0 \%$ & $39.4 \%$ & $55.9 \%$ \\
\hline Number of previous psychiatric hospitals & $0.73(0.93)$ & $0.64(.87)$ & $.92(1.01)$ \\
\hline Prior medication use (\%) & $55.5 \%$ & $51.9 \%$ & $62.5 \%$ \\
\hline Age at first psychosis & $11.13(3.48)$ & $11.40(3.29)$ & $10.54(3.85)$ \\
\hline Duration of Illness (mo) & $37.60(30.09)$ & $34.73(27.69)$ & $43.82(34.33)$ \\
\hline PANSS positive & $26.46(5.75)$ & $26.63(5.52)$ & $26.11(6.25)$ \\
\hline PANSS negative ${ }^{*}$ & $25.17(8.18)$ & $26.35(8.56)$ & $22.71(6.77)$ \\
\hline PANSS summary & $101.56(20.23)$ & $103.38(20.70)$ & $97.76(19.09)$ \\
\hline CGI & $5.61(0.87)$ & $5.70(0.88)$ & $5.45(0.82)$ \\
\hline BPRS-C & $42.96(11.40)$ & $42.98(11.68)$ & $42.92(10.97)$ \\
\hline $\begin{array}{l}\text { ote: } \text { BPRS-C }=\text { Brief Psychiatric Rating S } \\
\text { cale. }\end{array}$ & e for Children; CGI = Clin & ical Global Impression Scal & e; PANSS = Positive and \\
\hline
\end{tabular}

J Am Acad Child Adolesc Psychiatry. Author manuscript; available in PMC 2015 May 28. 


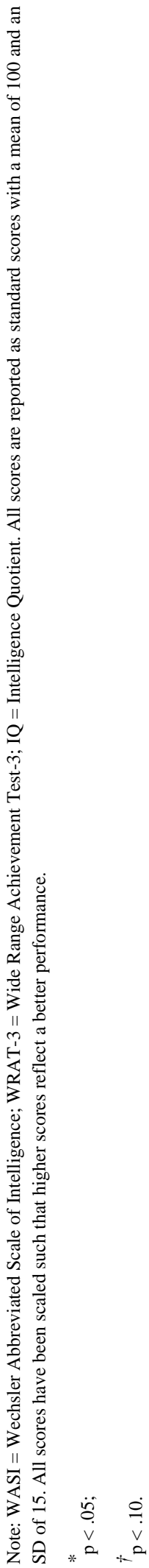

J Am Acad Child Adolesc Psychiatry. Author manuscript; available in PMC 2015 May 28. 


\section{TABLE 3}

Group Comparisons of Neurocognitive Variables, Controlling for Chronological Age

\begin{tabular}{|lrccc|}
\hline Domain & Total N & Schizophrenia & Schizoaffective & $\boldsymbol{F}$ Value \\
\hline Fine Motor Speed & $\mathbf{1 0 5}$ & $\mathbf{0 . 0 1}(\mathbf{0 . 8 2})$ & $\mathbf{- 0 . 0 6}(\mathbf{0 . 8 2})$ &. $\mathbf{0 7}$ \\
Fingertapping-Dominant Hand & 91 & $0.12(1.02)$ & $-0.23(.93)$ & 2.36 \\
Fingertapping-Nondominant Hand & 89 & $0.09(1.04)$ & $-0.15(.92)$ & .97 \\
Grooved Pegboard-Dominant Hand & 97 & $-0.00(1.00)$ & $0.01(1.01)$ & .05 \\
Grooved Pegboard-Nondominant Hand & 97 & $-0.05(1.01)$ & $0.08(1.00)$ & .65 \\
Attention & $\mathbf{9 9}$ & $\mathbf{0 . 0 6}(\mathbf{0 . 8 8})$ & $\mathbf{- 0 . 0 8}(\mathbf{0 . 8 6})$ & $\mathbf{. 3 9}$ \\
Visual CPT Omissions & 91 & $0.04(0.89)$ & $-0.09(1.21)$ & .31 \\
Auditory CPT Omissions & 89 & $0.03(1.12)$ & $-0.05(0.77)$ & .004 \\
Working Memory & $\mathbf{1 0 6}$ & $\mathbf{- 0 . 0 9}(\mathbf{0 . 8 4})$ & $\mathbf{0 . 1 3}(\mathbf{0 . 7 4})$ & $\mathbf{2 . 0 8}$ \\
WJ-III Numbers Reversed Total & 93 & $-0.07(1.04)$ & $0.12(0.92)$ & 1.64 \\
Hopkins Verbal Learning Total & 98 & $-0.02(0.93)$ & $0.03(1.13)$ & .14 \\
Visual-Spatial Working Memory (5'Delay) & 81 & $-0.13(1.17)$ & $0.21(0.61)$ & $3.04 \dagger$ \\
WRAML Visual Learning Total & 96 & $-0.08(0.95)$ & $0.13(1.08)$ & .66 \\
Problem Solving Efficiency & $\mathbf{1 0 7}$ & $\mathbf{- 0 . 0 4}(\mathbf{0 . 8 7})$ & $\mathbf{- 0 . 0 4}(\mathbf{0 . 7 9})$ & $\mathbf{. 0 3}$ \\
Wisconsin Card Sort Categories & 90 & $-0.04(1.02)$ & $0.06(0.97)$ & .55 \\
Wisconsin Card Sort Perseverations & 90 & $-0.01(0.93)$ & $0.01(1.12)$ & .11 \\
Ruff Figural Fluency Unique Total & 95 & $0.01(1.05)$ & $-0.02(0.93)$ & .03 \\
COWA Semantic Fluency Total & 98 & $0.06(1.01)$ & $-0.10(0.99)$ & .47 \\
Inhibitory Control & $\mathbf{9 9}$ & $\mathbf{- 0 . 0 0 ( 1 . 0 1 )}$ & $\mathbf{- 0 . 0 3 ( 0 . 7 0 )}$ & $\mathbf{0 . 0 0 3}$ \\
Visual CPT Commissions & 91 & $0.03(0.97)$ & $-0.06(1.08)$ & .14 \\
Auditory CPT Commissions & 89 & $-0.01(1.15)$ & $0.02(0.66)$ & .10 \\
Social Cognition/Eyes Test & $\mathbf{9 4}$ & $\mathbf{0 . 0 4}(\mathbf{1 . 0 4})$ & $\mathbf{- 0 . 0 6}(\mathbf{0 . 9 5})$ & $\mathbf{. 1 7}$ \\
\hline
\end{tabular}

Note: $\mathrm{CPT}=$ Continuous Performance Test; WJ-III $=$ Woodcock-Johnson Test of Cognitive Abilities Third Edition; WRAML $=$ Wide Range Assessment of Memory and Learning; COWA = Controlled Oral Word Association Test. All scores are reported in Z-scores derived from the raw scores for the tests from the total sample. All scores have been scaled such that higher scores reflect a better performance.

$t_{\mathrm{p}}<.10$. 
TABLE 4

Partial Correlations Between the Neurocognitive Domains and Vineland Adaptive Behavior Scale

\begin{tabular}{|lllll|}
\hline Neurocognitive Domains & Communication & Daily Living Skills & Social & Adaptive Composite \\
\hline Fine-Motor Speed & 0.16 & $0.22^{*}$ & $0.24^{* *}$ & $0.25^{* *}$ \\
Attention & $0.37^{* * *}$ & $0.41^{* * *}$ & $0.33^{* * *}$ & $0.42^{* * *}$ \\
Working Memory & $0.45^{* * *}$ & $0.28^{* *}$ & $0.42^{* * *}$ & $0.44^{* * *}$ \\
Problem Solving Efficiency & $0.40^{* * *}$ & $0.26^{* *}$ & $0.44^{* * *}$ & $0.41^{* * *}$ \\
Inhibitory Control & $0.23^{*}$ & 0.12 & 0.14 & $0.19^{\S}$ \\
Social Cognition & $0.21^{*}$ & 0.17 & $0.27^{* *}$ & $0.23^{*}$ \\
Full-4 IQ & $0.39^{* * *}$ & $0.22^{*}$ & $0.38^{* * *}$ & $0.39^{* * *}$ \\
\hline
\end{tabular}

p $<.05$;

** $\mathrm{p}<.01$

*** $\mathrm{p}<.001 ;$

$\S_{\mathrm{p}}<.07$. 